\title{
Cálculo da Idade Gestacional: uma comparação entre a DUM, Capurro e USG
}

\author{
Gestational Age Estimation Method: a comparison between LMP, Capurro and USG \\ Cálculo de la Edad Gestacional: una comparación entre LMP, Capurro y USG
}

Gabriela Campos de Freitas Ferreira ${ }^{1 *}$, Andressa Tavares Parente ${ }^{1,2}$, Anna Paula Alves de Almeida$^{1}$, Juliana Santos de Albuquerque ${ }^{1}$, Rennan Coelho Bastos ${ }^{1}$, Franciane do Socorro Rodrigues Gomes ${ }^{1}$, Elannira Amaral Soares ${ }^{1}$, Angeline do Nascimento Parente ${ }^{1}$, Débora Talitha Neri $^{1}$, Antonio Claudio do Rêgo Coelho ${ }^{1,2}$.

\section{RESUMO}

Objetivo: Descrever o perfil sociodemográfico e obstétrico de puérperas e comparar os métodos de cálculo da idade gestacional (IG) dos recém-nascidos caracterizados em um hospital de referência materno-infantil na região Norte. Métodos: Estudo exploratório, descritivo transversal de natureza quantitativa, composto por 100 puérperas e seus respectivos recém-nascidos. Realizado em uma maternidade no período de julho a novembro de 2019. Os dados foram obtidos por formulários a partir dos prontuários, analisados por estatística descritiva com auxílio do software BioEstat 5.3. Resultados: A média de idade das puérperas foi 24 anos, $90 \%$ eram pardas/pretas e a metade moram na capital Belém (50\%). Todas as gestações foram únicas e 96\% delas realizaram pré-natal. $53 \%$ mantinham união estável e a maioria (26\%) cursava o ensino médio. A média da IG pelo Capurro foi de 39 semanas e 3 dias, pelo dia da última menstruação foi 39 semanas e pelo Ultrassonografia 38 semanas e 4 dias. Os recém-nascidos pesaram em média 3095,6 g, com índice de Apgar $>7$ no $1^{\circ}$ e ao $5^{\circ}$ minuto e estatura $<50 \mathrm{~cm}$. Conclusão: A determinação da idade gestacional por diferentes métodos apresentou como maior diferença sete dias entre USG e Capurro.

Palavras-chave: Idade gestacional, Enfermagem obstétrica, Perfil de saúde, Recém-nascido.

\begin{abstract}
Objective: To describe the social-demographic and obstetrics characteristics of puerperal patients and to compare the methods for calculating the gestational age (GA) of newborns assessed in a maternal and child care reference hospital in Northern Brazil. Methods: This is an exploratory, descriptive and cross-sectional study with a quantitative approach, composed of 100 mothers and their respective newborns. The study was held in a maternity hospital from July to November 2019. The data were obtained through forms filled with information collected in the medical records, analyzed using descriptive statistics and the BioEstat 5.3 software. Results: The average age of the puerperal women was 24 years old, $90 \%$ were black or brownskinned and half of them lived in the capital city Belém (PA) (50\%). All pregnancies were single gestations and $96 \%$ of them received prenatal care. $53 \%$ were in a cohabitating relationship and the majority $(26 \%)$ attended high school. The mean GA using the capurro method was 39 weeks and 3 days, considering the day of the last menstruation, it was 39 weeks and based in the Ultrasonography, it was 38 weeks and 4 days. The newborns weighed an average of $3095.6 \mathrm{~g}$ with an Apgar score $>7$ in the $1 \mathrm{st}$ and 5 th minute and height $<50$ $\mathrm{cm}$. Conclusion: The determination of gestational age by different methods showed that the greatest difference was seven days between USG and Capurro.
\end{abstract}

Key words: Gestational age, Obstetric nursing, Health profile, Infant newborn.

1 Universidade Federal do Pará (UFPA), Belém - PA. *E-mail: freitas-gabi1@hotmail.com

2 Fundação Santa Casa de Misericórdia do Pará (FSCMP), Belém - PA.

SUBMETIDO EM: 3/2021

ACEITO EM: 3/2021

PUBLICADO EM: 4/2021 


\section{RESUMEN}

Objetivo: Comparar los métodos para el cálculo de la edad gestacional de los recién nacidos en un hospital de referencia maternoinfantil de la región Norte de Brasil. Métodos: Estudio exploratorio, descriptivo, transversal, de carácter cuantitativo, compuesto por 100 puérperas y sus recién nacidos. Realizado en un hospital de referencia maternoinfantil en 2019, datos obtenidos por formularios de historias clínicas, analizados por médio de estadística descriptiva con la ayuda del software BioEstat 5.3. Resultados: La media de edad de las puérperas fue de 24 años, 90\% eran mestizas / negras y la mitad vivía en la capital Belém-PA (50\%). Todos los embarazos fueron únicos y $96 \%$ de ellos habían recebido atención prenatal. 53\% vivían en una unión estable y la mayoría (26\%) estudió hasta la escuela secundaria. La media de la EG por medio de capurro método fue 39s 3d, para el FUM 39s y para el USG 38s 4d. Los recién nacidos pesaron una media de 3095,6 g con una puntuación de Apgar $>7$ in el $1^{\circ}$ y $5^{\circ}$ minuto y una estatura $<50 \mathrm{~cm}$. Conclusión: La determinación de la edad gestacional por diferentes métodos mostró que la mayor diferencia fue de siete días entre USG y Capurro.

Palabras clave: Edad gestacional, Enfermería obstétrica, Perfil de salud, Recién nacido.

\section{INTRODUÇÃO}

Em 2018, o Brasil teve aproximadamente 3 milhões de nascidos vivos. Deste total, somente na região Norte, foram mais de 300 mil nascimentos, sendo o estado do Pará campeão em números dentre os estados que compõe a região, com mais de 140 mil recém-nascidos. Aproximadamente $78 \%$ destes nascimentos ocorreram a termo (entre 37 e 41 semanas de gestação) (BRASIL, 2018). A Idade Gestacional (IG), juntamente com o índice de Apgar e o peso ao nascer, são variáveis determinantes para o sucesso neonatal e redução da mortalidade, demonstrando a vitalidade e maturidade dos conceptos (QUEIROZ MN, et al., 2018).

A IG no período gravídico, é determinada, prevalentemente, pela data da última menstruação (DUM) e ultrassonografia. Verificada em semanas ou dias inteiros, a IG é definida como o tempo decorrido a partir do primeiro dia do último ciclo menstrual regular. No entanto, a DUM relatada pode não ser um método totalmente confiável, pois a mulher pode não lembrar precisamente a data ou interpretar de maneira errada o sangramento precoce da gravidez como menstruação normal, além de existirem ciclos menstruais irregulares. Logo, existe um dilema acerca da DUM relatada para precisão da IG (SOUZA ABG, 2014).

A Ultrassonografia (USG) precoce, entre 10-13 semanas de gestação, é o método mais confiável para determinação da IG na gravidez, visto que neste período a taxa de variação do crescimento fetal é muito pequena. Entretanto, a estimativa da IG pela DUM, é o método recomendado pela Organização Mundial de Saúde (OMS), devido a sua alta acessibilidade e baixo custo, enquanto a USG precoce, muitas vezes, fica restrita a um grupo com maior acesso a recursos e a serviços de saúde (HOFFMAN CS, et al., 2008).

Após o nascimento, o exame físico do Recém-Nascido (RN), tem sido cada vez mais utilizado como estratégia complementar na definição da IG, não somente na ausência dos dados de ultrassom ou DUM. Apesar de ser amplamente utilizado na prática clínica, ainda existem poucos estudos avaliando a precisão do exame físico para medida da IG, porém, seu uso tem impacto direto na precisão do diagnóstico do estado antropométrico de um RN, e consequentemente, nas condições de saúde ao nascimento (NUNES MFP, et al., 2011). O método de exame físico para avaliação da IG do RN mais utilizado atualmente, é o de Capurro somático, que é determinado através de cinco paramentos físicos. A cada um dos parâmetros se atribui uma pontuação, e a somatória + 204/7 definirá a estimativa da IG (NUNES MFP, et al., 2011).

No contexto da temática e sua relevância para saúde neonatal, questionou-se: como apresenta-se o cálculo para idade gestacional em diferentes métodos de definição? Propôs-se a realização deste estudo, com o objetivo de descrever o perfil sociodemográfico e obstétrico das puérperas e comparar os métodos de cálculo da idade gestacional dos recém-nascidos caracterizados em um hospital de referência materno-infantil na região Norte. 


\section{MÉTODOS}

Realizou-se um estudo exploratório, descritivo de delineamento transversal e natureza quantitativa. Tratase de um recorte da pesquisa "Práticas obstétricas na assistência ao parto e nascimento em uma maternidade de referência". A amostra foi composta por 100 puérperas e seus respectivos recém-nascidos e foi realizada em um hospital de referência materno infantil em Belém, no estado do Pará, com período de coleta de julho a novembro de 2019.

Os critérios de inclusão foram puérperas de risco habitual maiores de 18 anos, que pariram recémnascidos com idade gestacional de 37 semanas ou mais, que entraram em trabalho de parto espontaneamente ou por indução, e pariram por via vaginal. Como critério de exclusão: puérperas menores de 18 anos, as que não apresentarem capacidade física e/ou emocional e ainda as que não aceitaram participar do estudo, além das que tiveram conceptos que evoluíram a óbito ou natimortos.

Os dados foram obtidos por meio de formulários preenchidos com base em informações disponíveis no prontuário das puérperas, incluindo dados gerais, idade gestacional pelo método DUM, USG e Capurro. A estimativa da IG pelo Capurro foi realizada por neonatologistas, conforme procedimento padrão da maternidade. A pesquisa mais ampla abrangeu 214 puérperas. O cálculo do tamanho da amostra foi realizado por meio da calculadora online netquest, a partir da quantidade de partos anuais, com intervalo de confiança de $90 \%$ e erro amostral de 5\%. Desse total (214), cerca de 100 mulheres estavam dentro do universo selecionado para este recorte.

As variáveis foram comparadas através de estatística descritiva: método DUM e USG para avaliação da idade gestacional, tendo como ponto de referência o método de Capurro somático. Os formulários foram codificados em siglas "P", inicial de "Puérpera", seguida de numeração sequenciada (P1, P2, P3 e, etc.), visando preservar o anonimato. Em sequência, os dados foram tabulados em planilhas do Microsoft Office Excel e submetidos a análise descritiva através do Programa BioEstat 5.3.

A pesquisa obedeceu a resolução n 466/2012 do Conselho Nacional de Saúde, do Ministério da Saúde, que traz as normas e diretrizes regulamentadoras das pesquisas envolvendo seres humanos quanto aos aspectos éticos (BRASIL, 2012). O projeto foi submetido ao Comitê de Ética em Pesquisa (CEP) da Fundação Santa Casa de Misericórdia do Pará, sendo aprovado sob Número do Parecer: 3.364.549, em junho de 2019.

\section{RESULTADOS}

A amostra foi composta por 100 mulheres. A Tabela 1 apresenta o perfil sociodemográfico e obstétrico das puérperas, com idades entre 18 e 40 anos, sendo a média de 24 anos. Em relação a cor/raça/etnia $91 \%$ se autodenominaram parda/morena/mulata ou preta. Quanto a procedência dessas mulheres, $50 \%$ são de Belém (capital) ou região Metropolitana, $46 \%$ de municípios do interior do estado do Pará.

Em relação ao estado civil, o estudo aponta que $63 \%$ das mulheres encontram-se em relação fixa (casada/união estável). Quanto a escolaridade, a pesquisa revelou que $26 \%$ apresentavam o ensino médio incompleto. A gestação única contemplou $100 \%$ da amostra e $96 \%$ das gestantes realizaram pré-natal, sendo a média do número de gestações de 1.3 e o registro máximo de 7 . A ocorrência de aborto foi descrita em $22 \%$ das participantes. Foram relatadas intercorrências gestacionais em $25 \%$ da amostra, sendo a maioria delas não especificada (Tabela 1). 
Tabela 1 - Perfil sociodemográfico e obstétrico das puérperas pesquisadas, $n=100$.

\begin{tabular}{|c|c|c|c|c|c|}
\hline Variável & Descrição & $\mathbf{N}$ & $\%$ & Média & $\begin{array}{l}\text { Desvio } \\
\text { padrão }\end{array}$ \\
\hline \multirow{3}{*}{ Idade (anos) } & $\leq 19$ & 18 & 18 & \multirow{3}{*}{24.6} & \multirow{3}{*}{5.6} \\
\hline & $20-34$ & 73 & 73 & & \\
\hline & $\geq 35$ & 9 & 9 & & \\
\hline \multirow{5}{*}{ Cor/raça/etnia } & Branca & 6 & 6 & \multirow{5}{*}{-} & \multirow{5}{*}{-} \\
\hline & Parda/Morena/Mulata & 84 & 84 & & \\
\hline & Amarela & 2 & 2 & & \\
\hline & Preta & 7 & 7 & & \\
\hline & Indígena & 1 & 1 & & \\
\hline \multirow{4}{*}{ Procedência } & Belém e região metropolitana & 50 & 50 & \multirow{4}{*}{-} & \multirow{4}{*}{-} \\
\hline & Outros municípios & 46 & 46 & & \\
\hline & Outros estados & 2 & 2 & & \\
\hline & Não informado & 2 & 2 & & \\
\hline \multirow{4}{*}{ Estado civil } & União estável & 53 & 53 & \multirow{4}{*}{-} & \multirow{4}{*}{-} \\
\hline & Solteira & 35 & 35 & & \\
\hline & Casada & 10 & 10 & & \\
\hline & Divorciada & 2 & 2 & & \\
\hline \multirow{7}{*}{ Escolaridade } & Ensino Fundamental Incompleto & 13 & 13 & \multirow{7}{*}{-} & \multirow{7}{*}{-} \\
\hline & Ensino Fundamental Completo & 2 & 2 & & \\
\hline & Ensino Médio Incompleto & 26 & 26 & & \\
\hline & Ensino Médio Completo & 24 & 24 & & \\
\hline & Ensino Superior Incompleto & 6 & 6 & & \\
\hline & Ensino Superior Completo & 5 & 5 & & \\
\hline & Não Informado & 24 & 24 & & \\
\hline Gestação & Única & 100 & 100 & - & - \\
\hline \multirow{2}{*}{ Pré-natal } & Sim & 96 & 96 & \multirow{2}{*}{-} & \multirow{2}{*}{-} \\
\hline & Não & 4 & 4 & & \\
\hline \multirow[b]{2}{*}{ Gesta } & 1 & 42 & 42 & \multirow{3}{*}{1.3} & \multirow{3}{*}{1.4} \\
\hline & $\begin{array}{l}\geq 2 \\
\text { Ḿ́xima } 7\end{array}$ & 58 & 58 & & \\
\hline \multirow{3}{*}{ Aborto } & $\begin{array}{c}\text { Máxima: } 7 \\
0\end{array}$ & & & & \\
\hline & $\geq 1$ & 78 & 78 & \multirow{2}{*}{0.3} & \multirow{2}{*}{0.7} \\
\hline & Máximo: 5 & 22 & 22 & & \\
\hline \multirow{3}{*}{ Parto } & 0 & 42 & 42 & \multirow{3}{*}{1} & \multirow{3}{*}{1.1} \\
\hline & $\begin{array}{l}\geq 1 \\
\text { Nã }\end{array}$ & $\begin{array}{l}42 \\
51\end{array}$ & 51 & & \\
\hline & $\begin{array}{l}\text { Não informado } \\
\text { Máximo: } 5\end{array}$ & 07 & 07 & & \\
\hline & Sim & 25 & 25 & & \\
\hline Intercorrências & Não & 74 & 74 & - & - \\
\hline & Não informado & 1 & 1 & & \\
\hline & Total & 100 & 100 & - & - \\
\hline
\end{tabular}

Fonte: Ferreira GCF, et al., 2020.

A descrição dos diferentes métodos para estimativa da idade gestacional contemplou média, mediana e desvio padrão da idade gestacional das puérperas: DUM, USG e Capurro. A média da idade gestacional estimada ao nascimento foi de 39 semanas de gestação para ambos os métodos: DUM e Capurro, sendo que a maior diferença entre as médias (7 dias) ocorrida entre USG e Capurro. A IG pela USG foi o dado com menor frequência na amostra: somente $74 \%$ possuía essa informação. $O$ menor desvio padrão foi registrado 
no método USG (1.2 semanas), bem como apresentou menor amplitude de cálculo da IG (36-42 semanas), semelhante ao Capurro (Tabela 2).

Tabela 2 - Média, mediana e desvio padrão das Idades Gestacionais pelos diferentes métodos: DUM, USG e Capurro, $n=100$.

\begin{tabular}{ccccccc}
\hline Método & IG (semanas) & $\mathbf{n}$ & $\%$ & Média* $^{*}$ & Mediana* $^{*}$ & $\begin{array}{c}\text { Desvio } \\
\text { padrão* }\end{array}$ \\
\hline Capurro somático & Mínima: 36 & 98 & 98 & 39s e 3d & 40 & 1.4 \\
$\begin{array}{c}\text { Máxima: } 42 \\
\text { MÚnima: 34 }\end{array}$ & 93 & 93 & 39s & 39 & 1.4 \\
USG & $\begin{array}{c}\text { Máxima: 43 } \\
\text { Mínima: 36 }\end{array}$ & 74 & 74 & 38s e 4d & 38 & 1.2 \\
\hline
\end{tabular}

Legenda: *semanas.

Fonte: Ferreira GCF, et al., 2020.

A Tabela 3 apresenta o perfil dos recém-nascidos. Quanto ao peso, a média total foi de $3.095 \mathrm{~g}$, com menor registro de $1.495 \mathrm{~g}$ e máximo de $4.008 \mathrm{~g}$. A maioria dos RN (58\%) pesaram mais de $2.500 \mathrm{~g}, 41 \%$ pesaram menos que $2.500 \mathrm{~g}$ e somente um registro com até $1.500 \mathrm{~g}$. Peso adequado para idade gestacional (AIG) foram $49 \%$ dos $\mathrm{RN}, 35 \%$ foram grandes para idade gestacional (GIG) e 16\% pequenos para idade gestacional (PIG).

O índice de Apgar no $1^{\circ}$ minuto foi maior que 7 em 92\%, menor ou igual a 7 ocorreu em $8 \%$. Já no $5^{\circ}$ minuto os números mostram que $93 \%$ teve Apgar de maior ou igual a 9 . Em relação ao comprimento, $60 \%$ dos RN mediram menos que $50 \mathrm{~cm}$ enquanto $40 \%$ ficaram entre 50 ou mais de estatura, o registro mínimo foi de $42 \mathrm{~cm}$ e máximo de $55 \mathrm{~cm}$, média de $49,8 \mathrm{~cm}$ (Tabela 3).

Tabela 3 - Perfil dos recém-nascidos pesquisados, $n=100$.

\begin{tabular}{ccccc}
\hline Variáveis & $\%(\mathbf{n})$ & Média & Min-máx & Desvio padrão \\
\hline Peso $(\mathbf{g})$ & $100(100)$ & 3095,6 & $1495-4008$ & 460,6 \\
$\geq 2.500 \mathrm{~g}$ & $58(58)$ & - & - & - \\
$<2.500 \mathrm{~g}$ & $41(41)$ & - & - & - \\
Até $1.500 \mathrm{~g}$ & $1(01)$ & - & - & - \\
GIG & $35(35)$ & - & - & - \\
AIG & $49(49)$ & - & - & - \\
PIG & $16(16)$ & - & - & 1,1 \\
\hline APGAR NO 10 & - & 8,4 & $3-9$ & - \\
\hline$>7$ & $92(92)$ & - & - & - \\
\hline 7 & $8(08)$ & - & - & - \\
\hline APGAR NO 50 & - & 9 & $6-10$ & - \\
\hline$\geq 9$ & $93(93)$ & - & - & 2,6 \\
$>7$ & $7(07)$ & - & - & - \\
\hline Estatura & - & 49,8 & $42-55$ & - \\
\hline > 50 cm & $40(40)$ & - & - & \\
\hline 50 cm & $60(60)$ & - & - & - \\
\hline
\end{tabular}

Fonte: Ferreira GCF, et al., 2020.

\section{DISCUSSÃO}

No que concerne a idade jovem das puérperas, os resultados obtidos foram semelhantes aos encontrados no estudo de Andrade SG, et al (2018), onde os autores traçaram o perfil de mulheres em uma maternidade no Ceará/Brasil, em que apontam a idade predominante de $20-30$ em 42,40\% da amostra. Segundo Brasil (2018), as mulheres entre $20-29$ foram responsáveis por quase $50 \%$ dos nascimentos vivos no ano, isso reforça este perfil nas maternidades. Pode-se constatar que, neste estudo há a prevalência mulheres do grupo de menor risco obstétrico, em idade fértil. 
A grande maioria das puérperas se autodeclararam pardas ou pretas (91\%) no estudo. Na Pesquisa Nascer no Brasil: Inquérito Nacional sobre Parto e Nascimento, $71 \%$ das mulheres também se autodeclaram quanto a cor da pele nessa variação, revelando que as mulheres pretas e pardas tendem a ser atendidas, em sua maioria, em hospitais da rede pública, nesse contexto, é importante destacar que diversos estudos apontam para as desigualdades raciais na atenção obstétrica (LEAL MC e GAMA SG, 2014).

No estudo de Leal MC, et al. (2017), foi evidenciado que as gestantes pretas sofrem maior risco de terem um pré-natal inadequado, falta de vinculação a maternidade, ausência de acompanhante e maior peregrinação para o parto, além de receberem menos informações sobre o início do trabalho de parto, possíveis intercorrências na gravidez e maior chance de nascimento pós termo. Gois EC, et al. (2019) investigaram que as mulheres pretas/pardas e solteiras estão no grupo de maior vulnerabilidade, apresentando baixos níveis de escolaridade e renda, bem como maiores dificuldades no acesso aos serviços de saúde.

Quanto a procedência dessas mulheres, a pequena diferença entre gestantes oriundas de Belém e região metropolitana para as vindas de municípios do interior do estado, revela que o perfil da maternidade em questão é de referência em obstetrícia, sendo amplamente divulgada e procurada por gestantes de todo o estado do Pará.

Considerando o estado civil, houve prevalência de relações com parceiro fixo em $63 \%$ (união estável e casadas), porém em um percentual menor do que foi evidenciado no estudo Andrade SG, et al. (2018): 95\%. Entretanto, foi maior do que o descrito por Brasil (2018,) que foi de pouco mais de $50 \%$. O fato de manter uma relação estável é um fator muito importante no ciclo gravídico puerperal, pois este é marcado por um turbilhão de emoções, sendo o suporte emocional essencial para o enfrentamento do processo de maternidade (DODT R, et al., 2010). A participação do pai/parceiro(a) durante todo o processo deve ser sempre estimulada, pois pode levar a melhora do bem-estar da mãe, bebê e dele próprio (ABREU LP, et al., 2019).

Com relação ao grau de instrução das puérperas, o estudo revelou que a maioria chegou a iniciar o ensino médio, porém não concluiu, revelando a dificuldade dessas mulheres, muitas vezes, de conciliar a maternidade aos estudos (Tabela 1). Puérperas com ensino médio incompleto ou completo corresponderam a $50 \%$ da amostra. Em 2018, quase $61 \%$ das mulheres que pariram conceptos vivos, estudaram até o ensino médio (BRASIL, 2018). A escolaridade das gestantes, segundo alguns autores, impacta diretamente na condição social, no acesso a melhores empregos e consequentemente na melhora da condição socioeconômica, o que pode ser determinante para quantidade de consultas de pré-natal, escolha da via de parto, aceitação do acompanhante e fornecimento de informações precisas durante as consultas, como a DUM, por exemplo (ANDRADE SG, et al., 2018).

Porém outro dado chama atenção: a elevada falta de informação sobre a escolaridade das puérperas, pois são dados que fornecem subsídios para identificação de lacunas sociais e o planejamento de ações para melhora nos indicadores de saúde. Diversos estudos revelam que a baixa escolaridade está relacionada a desfechos neonatais desfavoráveis e mortalidade materna e neonatal (HAIDAR FH, et al., 2001).

A não ocorrência de gestações múltiplas nas puérperas pesquisadas conforme Tabela 1, pode ser associada a prevalência de mulheres jovens, pois segundo Soares AMR, et.al. (2019), a incidência de gemelaridade aumenta com o avançar da idade materna, chegando a seu pico próximo aos 40 anos de vida, pois ocorre a máxima estimulação do Hormônio Folículo Estimulante (FSH), aumentando a taxa de desenvolvimento de folículos múltiplos. A reprodução assistida, a hiperestimulação ovariana e a fertilização in vitro são os principais fatores relacionados a gestações múltiplas.

Predominou entre as puérperas investigadas a realização do pré-natal: $96 \%$. Esse dado revela que a cobertura pré-natal se encontra cada vez mais universal, corroborando com os achados da Pesquisa Nascer no Brasil, onde 99\% das gestantes tinham cobertura da assistência pré-natal (LEAL MC e GAMA SG, 2014). A Rede Cegonha, criada através da Portaria GM/MS no 1.459/2011, vem fortalecer essa cobertura, estabelecendo direitos de planejamento reprodutivo, ampliação do acesso e melhoria da qualidade da atenção ao pré-natal, ao parto e ao puerpério e a assistência à criança até 24 meses, dessa forma, garantindo o 
acolhimento, as boas práticas e a segurança na atenção ao parto e nascimento, e aos aspectos do planejamento reprodutivo (BRASIL, 2011).

Mais de $50 \%$ das mulheres não eram primigestas e nem primíparas, em consonância com o estudo realizado por Leite FMC, et.al. (2013) em uma maternidade no Espírito Santo, onde $72,4 \%$ das puérperas tinham pelo menos uma gestação. Ainda nos registros da Tabela 1, 74\% das puérperas relataram não ter apresentado nenhum tipo de intercorrência durante a gestação, evidenciando e reforçando o perfil de baixo risco gestacional das mulheres pesquisadas.

A IG juntamente com o peso ao nascer são importantes indicadores de saúde neonatal, por isso, a acurácia do método de cálculo da idade gestacional é imprescindível. A DUM é o método comumente utilizado para mulheres com ciclo menstrual regular (entre 25 e 31 dias) e recomendado pela OMS. A USG do primeiro trimestre de gestação também é bastante utilizada, sendo mais precisa até a $20^{\text {a }}$ semana, podendo ser calculada a através das estruturas encontradas no útero até a $10^{\underline{a}}$ semana, bem como pela medida do comprimento do feto da cabeça às nádegas. Até a $20^{\underline{a}}$ semana medida do comprimento do fêmur ou do diâmetro biparietal é a mais adequada (SOUZA ABG, 2014).

O método Capurro somático, tanto na ausência como na presença de dados ultrassonográficos e DUM, vem sendo difundido e utilizado nas maternidades, tendo impacto direto na precisão no diagnóstico do estado antropométrico de um $\mathrm{RN}$ e, consequentemente, nas condições de saúde ao nascimento. O método é baseado na inspeção nas primeiras 12 horas de vida de sinais físicos que são colocados em uma escala de maturação, levando em consideração cinco características do recém-nascido: textura da pele, formato da orelha, tamanho do nódulo mamário, formação do mamilo e dobras da planta do pé e atribuindo pontos a essas características. Soma-se o total de pontos obtidos + 204 e divide-se por 7 (SOUZA ABG, 2014).

A partir da IG definida pela DUM, USG ou pelo método Capurro, os recém-nascidos podem ser classificados em: Pré-termo, <37 semanas e 0 dias; Termo: 37-42 semanas e 6 dias e Pós termo: $\geq 42$ semanas. O RN classificados como termo precoce, com idade gestacional ao nascimento entre 37 e 38 semanas, no limite da maturidade, têm maior risco de resultados adversos e mortalidade no período neonatal, comparados com os de 39-41 semanas. A IG é imprecisa em grande parte dos recém-nascidos, isso se deve em parte a, somente $45 \%$ das mulheres assistidas pelo Sistema Único de Saúde (SUS) terem acesso a USG obstétrica no primeiro trimestre da gestação, que é considerado o padrão ouro para estimativa da IG, visto que, há muitas dúvidas em relação a DUM (MIRALHA A, et al., 2017).

Na Tabela 2, os resultados revelam que a média entre os diferentes métodos de cálculo da IG não foram discrepantes, com diferença de dias de um método para o outro, bem como os valores extremos entre mínima e máxima registrados pelos diferentes métodos foram semelhantes pela USG e Capurro. O estudo realizado por Almeida MF, et al. (2008), observou que a concordância entre a IG calculada pela DUM vs USG<20 semanas foi menor do que em relação a comparação exame físico vs USG<20 semanas em recém-nascidos a termo, além da diferença entre a IG obtida pela DUM e USG<20 ser na maioria dos casos até uma semana, o que corrobora com resultados descritos na Tabela 2.

$\mathrm{Na}$ comparação entre os métodos, o estudo constatou que o Capurro somático superestimou a IG de maneira geral (média de 39 semanas e 3 dias), semelhante ao encontrado por Nunes MFP, et al. (2011) em sua pesquisa com $\mathrm{RN}$ a termo, em que teve como objetivo testar a acurácia dos métodos Capurro somático e Ultrassonografia, tendo como base a DUM, para avaliação da idade gestacional, sob diferentes métodos de análise e comparação estatística (Tabela 2).

A USG foi realizada em $74 \%$ da amostra, apontando que ainda é um recurso não acessível a todas as gestantes. Não houve registro nos prontuários do trimestre gestacional em que o exame foi realizado, o que pode explicar o menor desempenho na predição da IG no estudo e maior homogeneidade na amostra, pois 0 desvio padrão foi o menor. A pesquisa realizada por Guerreiro EM, et al. (2012) ratifica esta afirmação, com foco no pré-natal de uma Unidade Básica de Saúde (UBS) de Fortaleza/CE, onde foi constatado que as gestantes possuem muitas dificuldades na realização do exame ultrassonográfico obstétrico pelo SUS, por conta da demora, muitas vezes, elas são orientadas a procurar serviços privados, porém a realidade financeira de boa parcela delas não permite pagar pelo exame, o que dificulta a obtenção de uma USG precoce. 
O perfil dos recém-nascidos em Alojamento Conjunto (ALCON) obtido através da pesquisa, quanto ao peso, se assemelha ao encontrado por Sousa NMV, et al. (2018) no ALCON de uma maternidade pública no Triângulo Mineiro, onde ocorreu a prevalência de RN com peso adequados, sendo $88,3 \%$ com peso entre $2,500 \mathrm{~kg}$ a $4,000 \mathrm{~kg}$. Esses resultados coadunam aos apresentados pelo DATASUS no ano de 2018 , em que aproximadamente $80 \%$ (113.424) dos mais de 140.00 nascidos vivos no estado do Pará pesaram entre 2,500 $\mathrm{kg}$ e $3,999 \mathrm{~kg}$, bem como índice de Apgar igual ou maior que 8 do $1^{\circ}$ ao $5^{\circ}$ minuto de vida, corroborando também com os resultados apresentados na Tabela 3 (BRASIL, 2018).

\section{CONSIDERAÇÕES FINAIS}

A determinação da idade gestacional por diferentes métodos apresentou como maior diferença sete dias entre USG e Capurro. Constatou-se que o tema ainda é pouco explorado por pesquisadores, pelo reduzido número de trabalhos publicados tendo o assunto como foco. A pesquisa apresentou como limitação a quantidade amostral, que se mostrou pequena no decorrer de seu desenvolvimento, porém, os resultados encontrados nas variáveis analisadas estão em consonância com outros achados em diferentes regiões do país. Foi traçado o perfil sociodemográfico e obstétricos das puérperas e seus recém-nascidos atendidos na maior maternidade pública do Estado, e esses dados são de relevância, pois contribuem para a melhoria na qualidade da assistência prestada ao binômio mãe-bebê. Importante destacar que, ainda não há um consenso quando ao melhor método para estimar a idade gestacional, tendo cada um suas limitações. Todavia, USG do primeiro semestre continua uma referência para acompanhamento e definição da IG.

\section{REFERÊNCIAS}

1. ABREU LP, et al. Características obstétricas das gestantes submetidas à cesariana segundo a classificação de Robson. Revista Enfermagem Uerj, 2019; 27: 1-8.

2. ALMEIDA MF, et al. Comparação de medidas de idade gestacional. Universidade de São Paulo - Faculdade de saúde pública, 2008; 1: 1-20.

3. ANDRADE SG, et al. Perfil Sociodemográfico, Epidemiológico e Obstétrico de Parturientes em Um Hospital e Maternidade de Sobral. Revista Prevenção de Infecção e Saúde, 2018; 4: 7283.

4. AYRES M, et al. BioEstat 5.3: Aplicações estatísticas nas áreas das ciências biológicas e médicas, 2007; CNPQ.

5. BRASIL. DATASUS Tecnologia da Informação a Serviço do SUS. Base de dados TABNET. 2018.

6. BRASIL. Ministério da Saúde. Portaria № 1.459, DE 24 DE Junho de 2011. Institui, no âmbito do Sistema Único de Saúde - SUS - a Rede Cegonha. Brasília. Ministério da Saúde, 2011.

7. BRASIL. Ministério da Saúde. Resolução CNS 466/2012. Estabelece diretrizes e normas regulamentadoras de pesquisas envolvendo seres humanos. Brasília: Conselho Nacional de Saúde. Comissão Nacional de Ética em Pesquisa, 2012.

8. DODT R et al. Perfil epidemiológico de puérperas assistidas em um alojamento conjunto. Revista Enfermagem UERJ, 2010; 18(3): 345-351.

9. GOIS EC, et al. Mortalidade materna na Bahia no período de 2012 a 2016. Revista Eletrônica Acervo Saúde, 2019; 18: 335 1-8.

10. GUERREIRO EM, et al. O cuidado pré-natal na atenção básica de saúde sob o olhar de gestantes e enfermeiros. Revista Mineira de Enfermagem, 2012; 16(3): 315-323.

11. HAIDAR FH, et al. Escolaridade materna: correlação com os indicadores obstétricos. Cadernos de Saúde Pública, 2001; 17(4): 1025-1029.

12. HOFFMAN CS, et al. Comparison of gestational age at birth based on last menstrual period and ultrasound during the first trimester. Pediatric And Perinatal Epidemiology, 2008; 22(6): 587-596.

13. LEAL MC, et al. A cor da dor: iniquidades raciais na atenção pré-natal e ao parto no brasil. Cadernos de Saúde Pública, 2017; 33(1): 1-17.

14. LEAL MC, GAMA SGN. Nascer no Brasil. Cadernos de Saúde Pública, 2014; 30(1): 1-19.

15. LEITE FMC, et al. Perfil socioeconômico e obstétrico de puérperas assistidas em uma maternidade filantrópica. Cogitare Enfermagem, 2013; 18(2): 344-350.

16. MIRALHA A, et al. Prevenção da prematuridade - uma intervenção da gestão e da assistência. Departamento Científico de Neonatologia. Sociedade Brasileira de Pediatria, 2017; 2.

17. NUNES MFP, et al. Estimando a idade gestacional e sua relação com a antropometria status de recém-nascidos: a estudo comparando os métodos Capurro e ultrassom com a última menstruação período. Revista Brasileira de Saúde Materno Infantil, 2011; 11(1): 51-60.

18. QUEIROZ MN et al. Idade gestacional, índice de Apgar e peso ao nascer no desfecho de recém-nascidos prematuros. Com. Ciências Saúde, 2018; 29(4): 236-242.

19. SOARES AMR, et al. Complicações materno-fetais de gestações gemelares. Revista Caderno de Medicina, 2019; 2(1): 74-87.

20. SOUSA NMV, et al., Perfil das puérperas e neonatos assistidos em uma Maternidade pública da Região do Triângulo Mineiro. Revista Master, 2018; 3: 1-7.

21. SOUZA ABG. Enfermagem neonatal: cuidado integral ao recém-nascido. São Paulo: Atheneu, 2ª ed, $2014 ; 200$ p. 